\title{
Evaluación de la fertirrigación por goteo superficial y subsuperficial en el rendimiento de papa (Solanum tuberosum L.)
}

\author{
Evaluation of surface and subsurface drip \\ fertigation on potato yield (Solanum tuberosum L.)
}

José Lauro Conde Solano Universidad Técnica de Machala (Ecuador) jconde@utmachala.edu.ec

Edison Ramiro Vásquez

Universidad Nacional de Loja (Ecuador)

Revista Cumbres Vol.3 №2

Versión impresa ISSN 1390-9541

Versión electrónica ISSN 1390-3365

http://investigacion.utmachala.edu.ec/revistas/index.php/Cumbres 


\section{RESUMEN}

Con el objetivo de evaluar la disponibilidad de NPK a 10, 30 y $50 \mathrm{~cm}$ de profundidad con fertirrigación de NPK, suministrado por goteo superficial y subsuperficial a $25 \mathrm{~cm}$ de profundidad en el rendimiento del cultivo de papa (Solanum tuberosum L.), se instaló un experimento en diseño de bloques al azar con tres réplicas, en la Estación Experimental "La Argelia” de la Universidad Nacional de Loja. La frecuencia, tiempo y caudal de los emisores fueron similares para los tres tratamientos y se definieron en función de las lecturas del tensiómetro de mercurio. El mayor rendimiento $\left(16,97 \mathrm{TM}^{-1}\right)$ se obtuvo con riego por goteo subsuperficial y con fertilización; mientras que el menor rendimiento $\left(8,14 \mathrm{TM} \mathrm{ha}^{-1}\right)$ se obtuvo con el riego por goteo y sin fertilización. Referente a las concentraciones de NPK, las mayores concentraciones corresponden al riego superficial: 14,7\% de Nitrógeno, 56 \% de fósforo y $20 \%$ de potasio.

Palabras clave: Irrigación superficial, irrigación subsuperficial, concentración de NPK, fertirrigación.

\section{ABSTRACT}

With the aim of assessing the availability of NPK to 10, 30 and $50 \mathrm{~cm}$ depth with fertigation of NPK supplied by surface and subsurface drip to $25 \mathrm{~cm}$ depth in the yield of potato (Solanum tuberosum L.), an experiment in a randomized block design with three replicates, in the Experimental Station "Algeria" of the National University of Loja was set up. The frequency, time and flow rate of the issuers were similar for the three treatments and were defined on the basis of the readings of mercury. The higher performance (16.97 TM ha-1) was obtained with subsurface drip irrigation and fertilization; while the lower yield (8.14 $\mathrm{t} \mathrm{ha}^{-1}$ ) was obtained with drip irrigation and fertilization. With regard to the NPK concentrations, the highest concentrations correspond to the surface irrigation; nitrogen (14.7\%), phosphorous (56\%), and potassium (20\%).

Keywords: Surface irrigation, subsurface irrigation, concentration of NPK, fertigation.

\section{INTRODUCCIÓN}

La necesidad de suministrar alimentos a una creciente población del mundo, estimuló el interés de aumentar la eficiencia del riego y la fertilización, en razón que el recurso hídrico es cada vez más escaso y existe más competencia entre los diversos usos. El agua destinada para la agricultura representa el 70\% del agua extraída de pozos según Hossain et al. (2016). El riego por goteo subsuperficial y superficial puede reducir el uso del agua (Shock C. y Welch T. 2013). Para el Ecuador el aprovechamiento de agua en irrigación

\section{Cumbres}


constituye el $82 \%$ del consumo total (Zapata y Gasselin, 2005); esto obliga a utilizar eficientemente y con eficacia los sistemas de riego y la fertilización. En el caso del riego por goteo subsuperficial puede ahorrar hasta un $37 \%$ de agua frente al riego tradicional por gravedad según Montemayor-Trejo et al. (2012).

El hombre desde antes de la era cristiana, ya se ingenió técnicas de exploración y uso del agua especialmente en la agricultura. Estas técnicas han ido evolucionando con el paso del tiempo y la fertirrigación es una técnica moderna de aplicación de fertilizantes a los cultivos aprovechando la red de tuberías, el flujo del agua y la energía, especialmente en los sistemas de riego localizados como el goteo y la microaspersión (Kafkafi y Tarchitzky, 2012). La fertirrigación actualmente se ha generalizado en países como USA, Israel, Holanda, Italia, y España; mientras que en Suramérica y el Ecuador en particular va tomando cada vez mayor aceptación.

El riego por goteo subsuperficial es una alternativa válida, que se potencia por el mayor aprovechamiento de los nutrientes al ser entregados directamente a la zona de raíces. Al respecto Malcolm et al. (2008) señalaron que la temperatura de la zona de raíz influye en la asimilación y transpiración del melocotonero (durazno), lo que se traduce en un incremento de la eficiencia de riego, menor cantidad de agua con respecto al goteo superficial.

Con estas consideraciones, en la Estación Experimental "La Argelia" de la Universidad Nacional de Loja, se instaló un experimento con el objetivo de evaluar la disponibilidad de NPK a 10, 30 y $50 \mathrm{~cm}$ de profundidad con fertirrigación suministrado por goteo superficial y subsuperficial a $25 \mathrm{~cm}$ de profundidad y su influencia en el rendimiento del cultivo de papa (Solanum tuberosum L.). La elección del cultivo se basó fundamentalmente en su importancia en la dieta alimenticia a nivel mundial, llegando a considerarse como el tercer alimento de la población (FAO, 2008).

\section{MATERIALES Y MÉTODOS}

La investigación se desarrolló en el sector Los Molinos, de la Estación Experimental "La Argelia", de la Universidad Nacional de Loja, entre las coordenadas N: 9553240 m, E: 699900 m, y N: 9553200 m E: 699920 m. Zona Geográfica 17 S; altitud: 2138 msnm. Según Holdridge (1967) corresponde a una zona de vida de Bosque Seco Montano Bajo (bs-MB). Según Köppen (1936) pertenece a un clima templado lluvioso, mesotérmico, frío o isotermal.

Los principales materiales y equipos utilizados en la instalación del riego por goteo fueron: tuberías de polietileno, goteros tipo botón (vortex), filtros de malla, válvulas de control, tanque fertilizador y tensiómetros. La tubería principal fue de $32 \mathrm{~mm}$ y los laterales de riego de $16 \mathrm{~mm}$, el caudal de los goteros fue de 2,0 l/h e instalados a 0,50 m. El tanque fertilizador se ubicó a una altura piezométrica de 1,85 m, la altura entre el sitio del experimento y el reservorio fue de 2,0 m. 
El diseño experimental fue bloques al azar con tres réplicas y nueve unidades experimentales, los tratamientos corresponden: testigo (T1), que se suministró riego por goteo superficial sin fertilización; goteo superficial y fertilización (T2); y goteo subsuperficial a $25 \mathrm{~cm}$ de profundidad y fertilización (T3). La superficie por unidad experimental fue de $48 \mathrm{~m}^{2}$ (6m x $8 \mathrm{~m}$ ). Las variables investigadas fueron: rendimiento del cultivo en $\mathrm{kgha}^{-1} \mathrm{y}$ contenidos de NPK (Figura 1)

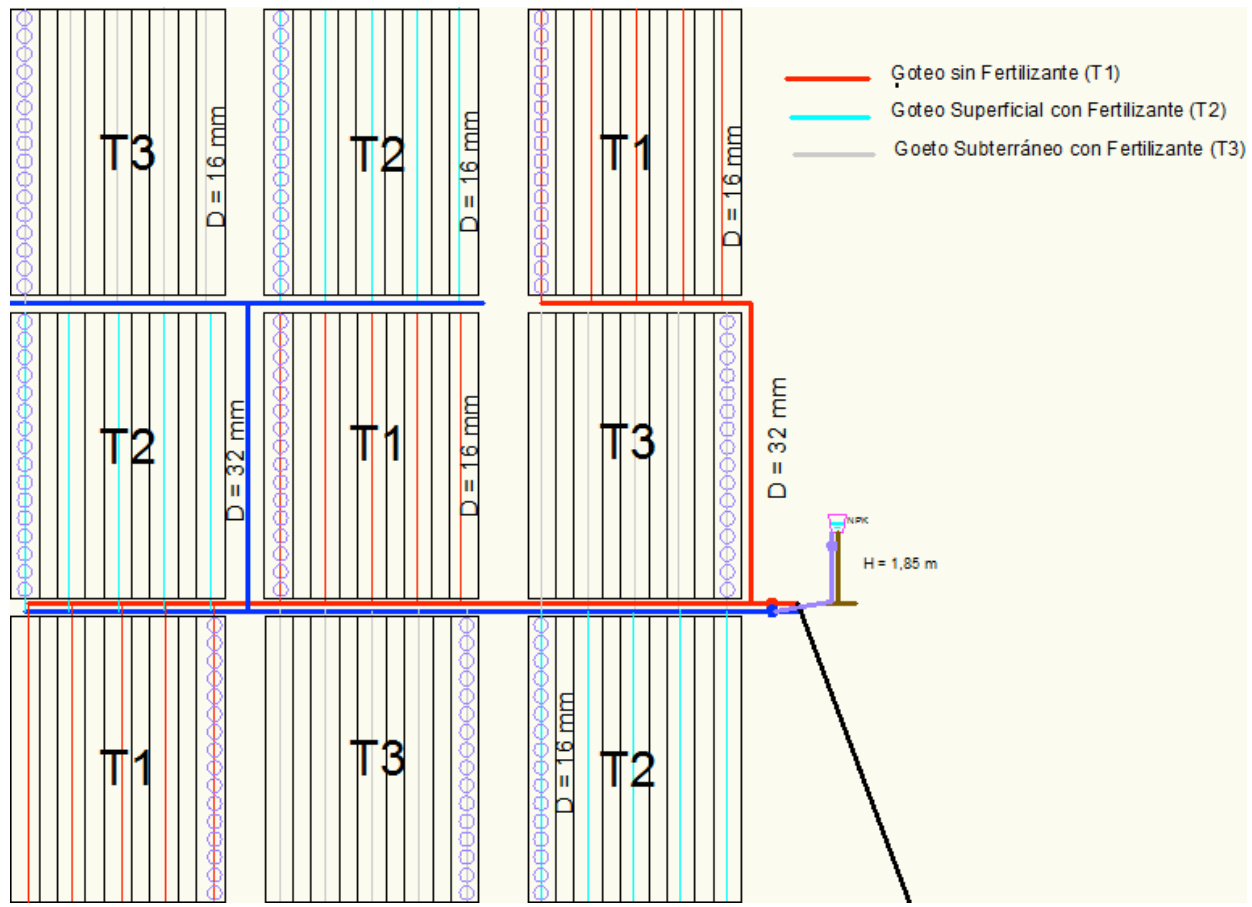

Fig. 1. Diseño del sistema de riego y distribución de los tratamientos en el campo experimental

Previo a la instalación del sistema de riego por goteo y la siembra, se enviaron muestras de agua para riego al laboratorio para su análisis químico; para el caso de la fertilidad del suelo (NPK) se realizó el análisis antes de la cosecha y después de la cosecha igualmente enviando muestras al laboratorio para su análisis. El nitrógeno del suelo se determinó mediante micro Kjeldahl, el fósforo a través de Olsen modificado por extracción con una solución alcalina de $\mathrm{pH}$ 8,5 y el potasio empleando también el método de Olsen. Los valores de N, P, y K, de la Tabla 1 son valores promedios de 10 y $30 \mathrm{~cm}$ de profundidad, y de sus respectivos tratamientos durante el ciclo del cultivo. En lo referente a la energía utilizada para el funcionamiento del tanque fertilizador se utilizó su propia energía piezométrica.

Para el análisis estadístico se pesaron los tubérculos de 10 plantas por surco, la producción se clasificó en: grandes, medianas y pequeñas.

Los resultados experimentales se procesaron con el software InfoStat mediante el modelo matemático del análisis de varianza de dosificación doble, para discriminar las medias se utilizó la prueba de Rangos Múltiples de Duncan con un nivel de significancia del 0,05.

\section{Cumbres}


Las dosificaciones fueron: $150 \mathrm{~kg} \mathrm{ha}^{-1}$ de $\mathrm{N} ; 80 \mathrm{~kg} \mathrm{ha}^{-1}$ de $\mathrm{P}_{2} \mathrm{O}_{5} ; \mathrm{y} 100 \mathrm{~kg}$ ha-1 de $\mathrm{K}_{2} \mathrm{O}$. La aplicación de la fertirrigación se realizó con: Úrea (46-0-0) para nitrógeno; Ácido fosfórico líquido (0-85-0) para fósforo; y Nitrato de potasio (13-0-46) para potasio. Las dosificaciones de los fertilizantes por cada riego fueron: $0,21 \mathrm{~kg}$ de Nitrato de potasio; 0,09 kg de acido fosfórico líquido; y $0,26 \mathrm{~kg}$ de urea. El volumen de agua que se utilizó estuvo en función de la solubilidad del producto

\section{RESULTADOS Y DISCUSIÓN}

\section{Programación del riego}

La programación del riego se realizó a través del tensiómetro, para esto se obtuvo la curva de calibración del tensiómetro en función del contenido de humedad del suelo.

\section{Rendimiento de la producción del cultivo de papa}

Los rendimientos obtenidos de acuerdo a los tratamientos son: riego por goteo superficial sin fertilización, $48,84 \mathrm{~kg}\left(8,14 \mathrm{Tm} \mathrm{ha}^{-1}\right)$; riego por goteo superficial con fertirrigación, 86,65 kg (14,44 $\left.\mathrm{Tm} \mathrm{ha}^{-1}\right)$ y el rendimiento del cultivo irrigado por goteo subsuperficial con fertirrigación, 101,82 kg (16,97 $\mathrm{Tm} \mathrm{ha}^{-1}$ ). Los resultados del rendimiento de papa indican que el tratamiento irrigado por goteo subsuperficial fue mayor en $52 \%$ con respecto al testigo y en $14,9 \%$ con respecto al cultivo irrigado por goteo superficial; esto concuerda con lo encontrado por Tanaskovik, V., Cukaliev, O., Romić, D., \& Ondrašek, G. (2011) que obtuvieron un rendimiento de 39\% mayor de tomate irrigado por goteo con respecto al irrigado por gravedad.

Los rendimientos guardan relación con los reportados por los investigadores de la empresa de riego NaanDanJainIberica (Fandiño, Martínez, Rey y Cancela, 2013) al expresar que con el riego por goteo subsuperficial la planta aprovecha mejor el Nitrógeno, Fósforo, y Potasio, lo que da como resultado un incremento de la cosecha y menor gasto de agua en el riego. Estos antecedentes guardan relación con lo afirmado por Lloveras et al. (2013), que plantearon que la correcta nutrición de las plantas depende del nitrógeno mineral presente en cada momento en el suelo; el mismo criterio tiene Rodríguez G, y, López T. (2014) que menciona que a profundidades entre 0 y 45 $\mathrm{cm}$, la raíz aprovecha mejor la reserva de agua y por lo tanto los nutrientes.

Además, los resultados guardan correspondencia con lo observado por Montesinos W. (2015) en cultivo de calabaza, donde se obtuvo mejor rendimiento con el riego por goteo subsuperficial. En el Instituto Nacional de Investigadores Forestales Agrícolas y Pecuarios de México (INIFAP, 2006) determinaron rendimientos máximos de maíz a través del riego subsuperficial.

\section{Disponibilidad de nitrógeno $(\mathrm{N})$, fósforo $(\mathrm{P})$ y potasio $(\mathrm{K})$}

Los resultados de disponibilidad de NPK (tabla 1) indicaron que las unidades experimentales irrigadas con riego subsuperficial mostraron menos nitrógeno (15\%), menos fósforo (52\%) y menos potasio (20\%) con respecto al riego 
superficial; mientras que el rendimiento de tubérculos fue mayor con el riego subsuperficial. Para este análisis se tomaron los valores de NPK a $10 \mathrm{~cm}$ de profundidad para el riego superficial, y $30 \mathrm{~cm}$ para el riego subsuperficial. El instituto internacional de nutrición de plantas indicó que las aplicaciones

Tabla 1. Contenidos de NPK (riego por goteo superficial y riego por goteo subsuperficial).

\begin{tabular}{|c|c|c|c|c|}
\hline TRATAMIENTO & $\begin{array}{c}\text { PROFUNDIDAD } \\
\text { (CM) }\end{array}$ & $\begin{array}{c}\mathbf{N} \\
\text { (PPM) }\end{array}$ & $\begin{array}{c}\mathbf{P}_{2} \mathbf{O}_{5} \\
(\mathbf{P P M})\end{array}$ & $\begin{array}{c}\mathbf{K}_{2} \mathbf{O} \\
(\mathbf{P P M})\end{array}$ \\
\hline $\begin{array}{c}\text { Riego por goteo } \\
\text { Superficial }\end{array}$ & 10 & 63,9 & 101,3 & 214,5 \\
\hline $\begin{array}{c}\text { Riego por goteo } \\
\text { Subsuperficial }\end{array}$ & 30 & 54,5 & 48,9 & 170,8 \\
\hline
\end{tabular}

continuas de $\mathrm{P}$ pueden estar disponibles varios años después de su aplicación (IPNI, 2013)

En el riego por goteo superficial no se evidenciaron diferencias al 5\% de significancia entre $10 \mathrm{~cm}$ y $30 \mathrm{~cm}$ de profundidad; mientras que con riego por goteo subsuperficial existió diferencia entre la concentración de $\mathrm{N}$ y $\mathrm{P}$, a excepción del K (Figura 2).

En el riego superficial las plantas no aprovecharon adecuadamente los fertilizantes, ya que se encontraron en mayor cantidad; mientras que en el riego subsuperficial fueron mejor aprovechados y se encontraron en menor cantidad. Estos resultados muestran concordancia con lo publicado por investigadores de la empresa israelí NaanDanJainIberica, que indicaron que el mayor aprovechamiento de fertilizantes ocurre cuando se aplica directamente en la zona radicular de la planta (Fandiño et al., 2013). Este criterio también es compartido por de la Cuesta, Á. y Alejandra, M. (2012).

A profundidades entre 10 y $30 \mathrm{~cm}$ existe un descenso en la concentración de NPK, respecto a una mayor disponibilidad de fósforo $\left(13,6 \mathrm{mg} \mathrm{kg}^{-1}\right)$ entre 0 y $20 \mathrm{~cm}$ de profundidad y 5,61 $\mathrm{mg} \mathrm{kg}^{-1}$ entre 20 y $40 \mathrm{~cm}$ de profundidad. Los resultados informados por Díaz et al (2008) también guardan relación con los resultados obtenidos.

Quezada-Roldan, G. y Bertsch-Hernández (2012) demostraron que los nu-

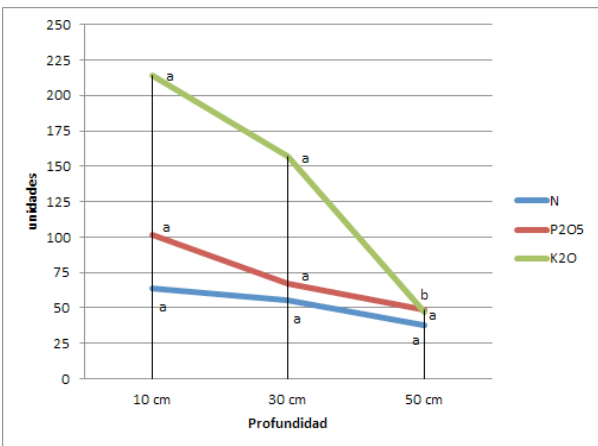

a

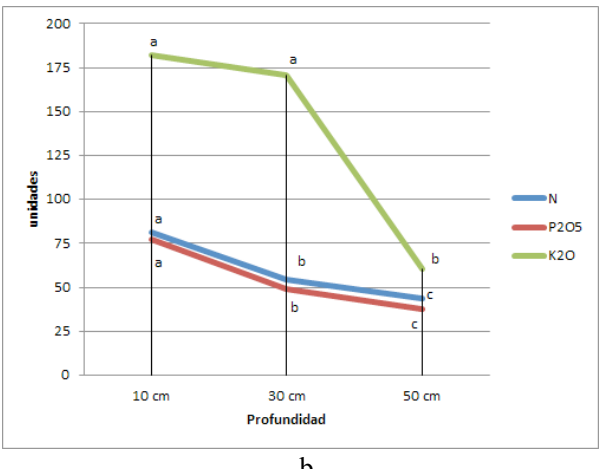

b

Fig. 2. Comportamiento de disponibilidad de N, P2O5 y K2O con la profundidad del suelo. a) para el tratamiento Riego superficial. b) para el tratamiento Riego subsuperficial

\section{Cumbres}


trientes suministrados en forma subsuperficial fueron mejor aprovechados por la planta.

\section{CONCLUSIONES}

El rendimiento del cultivo de papa irrigado con riego por goteo subsuperficial fue $14,9 \%$ mayor al riego por goteo superficial y $52 \%$ mayor al testigo. El nitrógeno, fósforo y potasio fueron mejor aprovechados por la planta, cuando la fertirrigación se aplicó en forma subsuperficial. Asimismo, se puede concluir que a mayor profundidad se encontró un descenso de disponibilidad de NPK, tanto en el riego por goteo superficial como en el subsuperficial.

\section{REFERENCIAS BIBLIOGRÁFICAS}

De la Cuesta, Á., Alejandra, M. (2012). Implementación de un sistema de fertirriego en un cultivo de cítricos (Doctoral dissertation, Corporación Universitaria Lasallista).

Fandiño, M., Martínez, E. M., Rey, B. J., \& Cancela, J. J. (2013). Valoración agronómica de la fertirrigación sobre Vitis vinifera cv. Mencía-DO Bierzo (España).

FAO. 2008, Review Año Internacional de la papa 2008.

Holddridge L. (1967). Clasificación de zonas de vida según el sistema de clasificación de Holdridge. San Jose. Centro Científico Tropical.

Hossain, S. A. A. M., Lixue, W., Uddin, M. E., Dan, L., Haisheng, L., \& Siping, L. (2017). Contemporary Perspective of Drip Irrigation: A Review of Water Saving Crop Production.

International Plant Nutrition Institute IPNI. (2013). A Manual for Improving the Management fo Plant Nutrition, Georgia-USA

Instituto Nacional de Investigaciones Forestales Agrícolas y Pecuarias INIFAP (2006). México. Revista Mexicana de Ciencias Agrícolas

Kafkafi, U., \& Tarchitzky, J. (2012). Fertirrigación: Una herramienta para una eficiente fertilización y manejo del agua. Asociación internacional de la industria de fertilizantes.

Köppen W. Clasificación Climática para el Ecuador, 1936

Lloveras Vilamanyà, J., Chocarro, C., \& Santiveri Morata, P. (2013). La fertilización de la alfalfa. Tierras de Castilla y León, 2013, núm. 200, p. 122-126.

Malcolm, P., Holford, P., McGlasson, B., \& Barchia, I. 2008. Leaf development, net assimilation and leaf nitrogen concentrations of five Prunus rootstocks in response to root temperature. Scientia horticulturae, 115(3), 285-291.

Montemayor-Trejo, J. A., Lara-Míreles, J. L., Woo-Reza, J. L., Munguía-López, J., Rivera-González, M., \& Trucíos-Caciano, R. (2012). Producción de maíz forrajero (Zea mays L.) en tres sistemas de irrigación en la Comarca Lagunera de Coahuila y Durango, México. Agrociencia, 46(3), 267-278.

Montesinos W. (2015). Comparación de la fertirrigación por goteo y microaspersión, en cultivo de calabaza, Tesis Ingeniero agrónomo, Universidad 
Autónoma Agraria Antonio Narro, Coahuila México.

Quesada-Roldán, G., \& Bertsch-Hernández, F. (2012). Fertirriego en el rendimiento de híbridos de tomate producidos en invernadero. Revista Agronomía Mesoamericana, 23(1).

Rodríguez García, M. R., \& López Seija, T. (2014). Comportamiento de la zona radical activa del banano en un Ferrasol bajo riego por goteo superficial y subsuperficial. Revista Ciencias Técnicas Agropecuarias, 23(3), 5-10.

Shock, C. C., \& Welch, T. (2013). El riego por goteo: Una introducción. Corvallis, Or.: Extension Service, Oregon State University.

Tanaskovik, V., Cukaliev, O., Romić, D., \& Ondrašek, G. (2011). The influence of drip fertigation on water use efficiency in tomato crop production. Agriculturae Conspectus Scientificus paper (ACS), 76(1), 57-63.

Zapatta, A., \& Gasselin, P. (2005). El riego en el Ecuador: problemática, debate y políticas. 